\title{
Brain abscess due to Aggregatibacter aphrophilus and Bacteroides uniformis
}

\author{
Maja Bogdan ${ }^{1^{*}}$, Vlasta Zujić Atalić ${ }^{1}$, Ivan Hećimoviće ${ }^{2,3}$, Dubravka Vuković ${ }^{1}$ \\ ${ }^{1}$ Institute of Public Health for the \\ Osijek-Baranja County, Department of \\ Microbiology, Osijek, Croatia, ${ }^{2}$ University \\ Hospital Center Osijek, Department of \\ Neurosurgery, Osijek, Croatia, ${ }^{3}$ University \\ "J. J. Strossmayer", School of Medicine \\ Department of Neurosurgery, Osijek \\ Croatia \\ ${ }^{*}$ Corresponding author: \\ maja.bogdan7@gmail.com \\ Tel.: + 38531225772 \\ Fax.: + 38531206870 \\ Received: 5 August 2015 \\ Accepted: 23 September 2015 \\ Key words: Child - Haemophilus \\ - Oropharynx - Odontogenic origin. \\ Objective. The aim of this report was to describe the occurrence of a \\ bacterial brain abscess in a healthy individual, without any predispos- \\ ing condition. Case report. A thirteen-year old boy was admitted to \\ the Department of Neurosurgery after the onset of vomiting, headache \\ and dizziness. A neurological deficit was detected during the physi- \\ cal examination so urgent magnetic resonance imaging of the brain \\ was performed, revealing an intrahemispheric, right positioned soli- \\ tary expansive mass with ring enhancement. Purulent material was \\ obtained during osteoplastic craniotomy with total extirpation of the \\ brain abscess. Aggregatibacter aphrophilus and Bacteroides uniformis \\ were isolated. The patient's general condition improved and the neu- \\ rological deficit subsided as a result of the prompt recognition and \\ treatment of this life threatening condition. Conclusion. To achieve \\ a favourable clinical outcome, prompt recognition and surgical treat- \\ ment of a brain abscess are of primary importance,followed by admin- \\ istration of appropriate antimicrobial therapy. To our best knowledge, \\ this is the first report of this combination of microorganisms as the \\ cause of a brain abscess.
}

\section{Introduction}

A bacterial brain abscess is a relatively uncommon but extremely serious and life threatening infection. The incidence ranges from $0.3-1.3 / 100,000$ persons per year, with around $2 \%$ associated with dental infections $(1,2)$. Intracranial abscesses can originate from infection of contiguous structures (e.g., otitis media, dental infection, mastoiditis, sinusitis), secondary to haematogenous dissemination from a remote site (especially in patients with cyanotic congenital heart disease), after skull trauma or surgery, and, rarely, following meningitis. In at least 15\% of cases, no source can be identified (3). Aggregatibacter aphrophilus (formerly Hae- mophilus aphrophilus) is a member of the normal flora of the human oral cavity and pharynx. It may cause brain abscess and infective endocarditis, and has been isolated from various other body sites, including the peritoneum, pleura, wounds and bone (4). Bacteroides spp. is generally isolated from mixed infections with other aerobic and anaerobic bacteria, forming a polymicrobial infection. Colonization of the oropharyngeal cavity can lead to the isolation of these species from brain abscesses (5).

The aim of this report was to describe for the first time the occurrence of a bacterial brain abscess due to this combination of microorganisms in a healthy child without any predisposing condition. 


\section{Case report}

A healthy thirteen-old-boy was admitted to the Department of Neurosurgery at the University Hospital Centre, Osijek, due to frontally positioned headache, dizziness and vomiting. The patient was conscious, and physical examination revealed mild left hemiparesis, driftage during walking and instability in the Romberg position without signs of meningism. Terminal left diplopia and inability of terminal abduction of left bulbus were also detected during the examination of his bulbomotoric abilities. Five days before admission to the hospital, he had had a case of diarrhoea with headache, dizziness and vomiting, and was subfebrile $\left(37.5^{\circ} \mathrm{C}\right.$ axillary measurement). He was examined by an infectious disease specialist who recommended a dietary regimen and rehydration at home. The headache did not subside and was even enhanced during movement, so urgent magnetic resonance imaging (MRI) of the brain was performed and revealed an intrahemispheric, right positioned, solitary expansive mass, diameter $41 \times 31 \mathrm{~mm}$, characteristically ring shaped with peripherally contrast enhancing, peripheral oedema and mass effect to the left (Figure 1).
Cardiovascular, respiratory and abdominal examinations revealed no abnormalities. Laboratory findings were: C-reactive protein $0.5 \mathrm{mg} / \mathrm{l}$, erythrocyte sedimentation rate 18 $\mathrm{mm} /$ hour, and white blood cell count 6.6 x $10 \% / 1$. Only dental problems (caries) were detected. Prior dental treatment was excluded. Paediatric HIV infection is very rare in Croatia. In the period of 1985-2014 only 14 HIV positive children from HIV positive mothers were reported (6). Therefore we did not regard HIV testing to be necessary. The right parietal osteoplastic craniotomy was performed with complete evacuation of the purulent material and the abscess capsule. Empirical therapy with ceftriaxone and metronidazole was started. Samples obtained during the operation were immediately sent to a microbiology laboratory for aerobic and anaerobic cultivation. Blood agar plate and Brain-Heart infusion broth were inoculated and incubated aerobically at $37^{\circ} \mathrm{C} / 24 \mathrm{~h}$. Chocolate agar plate after inoculation was incubated at $37{ }^{\circ} \mathrm{C}$ in atmosphere with 5\%$10 \% \mathrm{CO}_{2}$. Columbia agar and thioglycolate broth were also inoculated and incubated at $37{ }^{\circ} \mathrm{C} / 48 \mathrm{~h}$ in anaerobic atmosphere. Direct sample smear revealed Gram-negative bacilli with heavy polymorfonuclear infil-

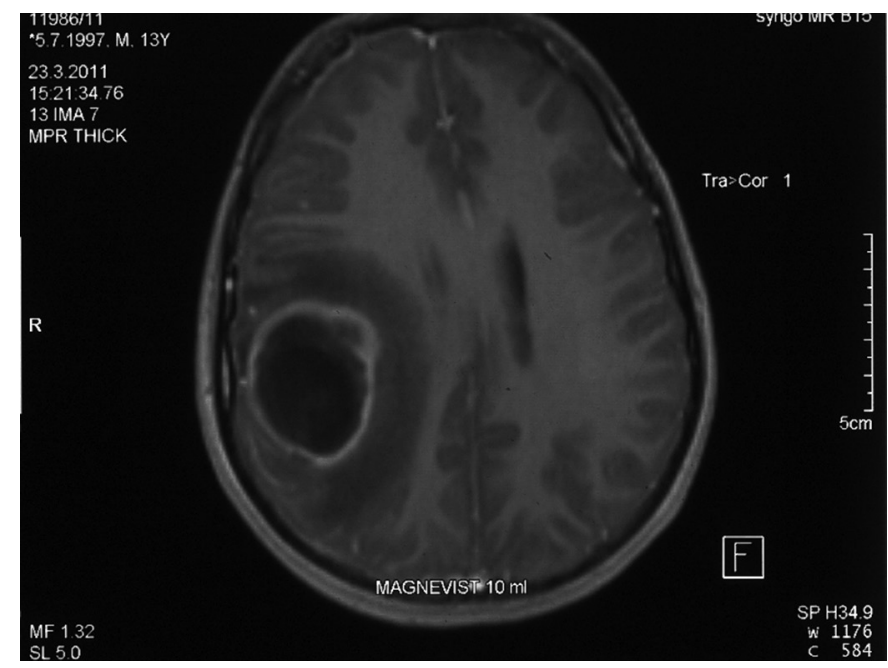

Figure 1 Characteristically ring shaped right positioned, intrahemispheric solitary expansive mass, with peripherally contrast enhancing and peripheral oedema. 
tration. On the chocolate agar opaque, yellowish, catalase, urease and oxidase negative colonies of Gram-negative bacilli grew, identified by API NH system (bioMérieux, Marcy l'Etoile, France) as Haemophilus aphrophilus. Antimicrobial susceptibility was detected by the Kirby- Bauer disc diffusion method and the isolate was susceptible to ampicillin, amoxicillin-clavulonic acid, sulfametoxasole-thrimetoprime, ceftriaxone and meropenem, according to EUCAST breakpoint standards (7). From the material cultivated in anaerobic condition whitish colonies of non-spore forming Gram-negative bacilli were isolated. Using API 20 A system (bioMérieux, Marcy l'Etoile, France) colonies were identified as Bacteroides uniformis, and for antimicrobial susceptibility testing purposes $\mathrm{ATB}^{\circledR} \mathrm{ANA}$ (bioMérieux, Marcy l'Etoile, France) was used. The strain was resistant to penicillin, clindamycin and amoxicillin, and susceptible to metronidazole, imipenem, amoxicillin-clavulonic acid, piperacillin-tazobactam and cefotaxime.

Metronidazole 4x250 mg and ceftriaxone $2 \times 2$ g were administered for sixteendays with a switch to cefixime $1 \times 400 \mathrm{mg}$ for ten days, on the recommendation of the infectious disease specialist. Control head MRI revealed substantial brain oedema regression, with minimal left ventricle compression. There was no mass effect to the left ventricle or signs of abscess residua. After the surgical drainage and antimicrobial therapy, the patient's general condition improved, with complete regression of discrete neurological deficit. Fifteen-month follow-up showed no signs of recurrence of the abscess.

\section{Discussion}

Haemophilus aphrophilus is one of the normal oral cavity flora. It is a fastidious Gramnegative bacillus part of the HACEK group, which includes Haemophilus species, $A g$ gregatibacter actinomycetemcomitans (for- merly Actinobacillus actinomycetemcomitans), Aggregatibacter aphrophilus (formerly Haemophilus aphrophilus and Haemophilus paraphrophilus), Cardiobacterium hominis, Eikenella corrodens and Kingella species involved in the cases of bacterial endocarditis with or without predisposing heart disease $(8,9)$. The species Haemophilus aphrophilus and Haemophilus paraphrophilus were reclassified as single species Aggregatibacter aphrophilus. They are Gram-negative, short regular bacilli, $0.5 \times 1.5-1.7 \mu \mathrm{m}$ with occasional filamentous forms. They require $5 \%-10 \% \mathrm{CO}_{2}$ for primary isolation. Their growth may be enhanced by haemin, but Xfactor is not an absolute requirement. Some isolates require V-factor (formerly Haemophilus paraphrophilus), whilst others are $\mathrm{V}$-factor independent (formerly Haemophilus aphrophilus). The colonies on chocolate agar are opaque, granular and yellowish, catalase and urease negative, oxidase variable (4). Congenital heart disease and dental procedures have been described as potential predisposing factors for infection with this organism, which can be isolated from gingival scrapings, interdental material and dental plaque (10). It is described as a cause of brain abscesses, and can develop even in otherwise healthy individuals with dental problems (11).

Bacteroides spp. is a strict anaerobe, Gram-negative bacillus, and part of the normal gastrointestinal flora. It can also colonise the oral cavity of patients with poor oral hygiene or those who have received antimicrobial therapy, especially with $\beta$-lactam agents (6). It is an important pathogen of various infections in children. The colonisation of the oropharyngeal cavity can lead to the isolation of these species from paediatric infections that originate in this area, such as aspiration pneumonia, lung abscesses, chronic otitis media, brain abscesses and subcutaneous abscesses, or burns near the oral cavity (6). Bacteroides spp. is generally 
isolated from mixed infections with other aerobic and anaerobic bacteria, forming a polymicrobial infection (6). Organisms of the bacteroides group are often isolated from abscesses in the temporal lobe, although metastatic, haematogenous and post-traumatic abscesses can be found in different sites (12). Brain abscesses are often polymicrobial, especially if they arise from a dental source (13). Most odontogenic brain abscesses occur following dental treatment, such as periodontal therapy and the extraction of infected teeth, as the integrity of the vascular endothelium is breached. The fact that dental procedures frequently cause bacteremia and extra oral infections is well documented. However, extra oral abscesses are rare because the body's immune system expunges most odontogenic bacteremia (14).

Appropriate management of mixed aerobic and anaerobic infections requires the administration of antimicrobials that are effective against both aerobic and anaerobic components of the infection, in addition to surgical correction and drainage of the pus. The environment of an abscess is detrimental for many antimicrobials. The abscess capsule interferes with the penetration of antimicrobial agents, and the low $\mathrm{pH}$ and the presence of binding proteins or inactivating enzymes may impair the activity of many antimicrobials (15). From samples obtained during the intraoperative removal of the brain abscess, Aggregatibacter aphrophilus and Bacteroides uniformis were isolated. This is the first time that this combination of microorganisms was isolated from a brain abscess in our microbiology department. Reviewing the literature, we did not find any reports of the same bacteria isolated from a brain abscess.

\section{Conclusion}

Since both bacteria can be found in the normal human oral cavity, as well as the patient's good general condition, without comorbidities, we believe that in this case the brain abscess originated from an odontogenic infection. It signifies the importance of preserving good oral hygiene, otherwise a serious infection may develop even in a healthy child. In conclusion, to achieve a favourable clinical outcome, prompt recognition and surgical treatment of the brain abscess are of primary importance, followed by administration of appropriate antimicrobial therapy.

Authors' contributions: Conception and design: $\mathrm{MB}$ and VZA; Acquisition analysis and interpretation of data: MB, VZA and IH; Drafting the article: $\mathrm{MB}$ and VZA; Revising it critically for important intellectual content: DV and IH.

Conflict of interest: The authors declare that they have no conflict of interest.

\section{References}

1. Ahamed SP, Lath S, DeGabriele GJ, Mathew VT. Cerebral abscess caused by Aggregatibacter aphrophilus. Neurosciences (Riyadh) 2010;15(1):40-2.

2. Tunkel AR. Brain abscess. In: Mandell GL, Bennett JE, Dolin R, editors. Mandell, Douglas, and Bennett's Principles and Practise of Infectuous diseases. 6th ed. Philadelphia (PA): Churhill Livingstone; 2006. p. 1150.

3. Mathisen GE, Johnson JP. Brain abscess. Clinical infectious diseases: an official publication of the Infectious Diseases Society of America. 1997;25(4):763-79; quiz 80-1.

4. Norskov-Lauritsen N, Kilian M. Reclassification of Actinobacillus actinomycetemcomitans, Haemophilus aphrophilus, Haemophilus paraphrophilus and Haemophilus segnis as Aggregatibacter actinomycetemcomitans gen. nov., comb. nov., Aggregatibacter aphrophilus comb. nov. and Aggregatibacter segnis comb. nov., and emended description of Aggregatibacter aphrophilus to include V factor-dependent and V factor-independent isolates. Int J Syst Evol Microbiol. 2006;56(Pt 9):2135-46.

5. Brook I. Bacteroides infections in children. J Med Microbiol. 1995;43(2):92-8.

6. Croatian Institute of Public Health. Croatian Health Service Yearbook 2014 [in Croatian]. Zagreb, 2015. [Updated 2015 May 12; Cited 2015 
Sep 9]. Available from: http://www.hzjz.hr/wpcontent/uploads/2015/12/ljetopis_2014.pdf

7. European Committee on Antimicrobial Susceptibility Testing. Breakpoint tables for interpretation of MICs and zone diameters. Version 1.1 April 2010. [Updated 2010 Apr 27; cited 2015 Aug 5]. Available from: http://www.eucast.org/fileadmin/ src/media/PDFs/EUCAST_files/Disk_test_documents/Eucast_breakpoints_v1.1.xls.

8. Winn WC Jr, Allen SD, Janda WM, Koneman EW, Procop GW, Schreckenberger PC, et al. Miscellaneous Fastidious Gram-Negative Bacilli. Color Atlas and Textbook of Diagnostic Microbiology. 6th ed. Philadelphia: Lippincot Williams and Wilkins; 2006.

9. Public Health England Identification of Haemophilus Species and the HACEK Group of Organisms, UK Standards for Microbiology Investigations. ID 12 Issue 3 2015. [Updated $2015 \mathrm{Feb}$ 3; cited 2015 Aug 5]. Available from: https://www. gov.uk/government/uploads/system/uploads/attachment_data/file/401404/ID_12i3.pdf.
10. Kao PT, Tseng HK, Su SC, Lee CM. Haemophilus aphrophilus brain abscess: a case report. J Microbiol Immunol Infect. 2002;35(3):184-6.

11. Tutuncu EE, Sencan I, Altay AF, Gurbuz Y. Brain abscess due to Haemophilus aphrophilus. Neurosciences (Riyadh). 2010;15(1):53-4.

12. de Louvois J, Gortavai P, Hurley R. Bacteriology of abscesses of the central nervous system: a multicentre prospective study. Br Med J. 1977;2(6093):981-4.

13. Simpson AJ, Das SS, Mitchelmore IJ. Polymicrobial brain abscess involving Haemophilus paraphrophilus and Actinomyces odontolyticus. Postgrad Med J. 1996;72(847):297-8.

14. Clifton TC, Kalamchi S. A case of odontogenic brain abscess arising from covert dental sepsis. Ann R Coll Surg Engl. 2012;94(1):e41-3.

15. Brook I. Microbiology of polymicrobial abscesses and implications for therapy. J Antimicrob Chemother. 2002;50(6):805-10. 